\title{
Incidence of complications following open mesh repair for inguinal hernia
}

\author{
Rashid Abd Elhalim Khalil ${ }^{1}$, Awad Ali M. Alawad ${ }^{2} *$ \\ ${ }^{2}$ Department of Surgery, Military Hospital, Kingdom of Saudi Arabia \\ ${ }^{1}$ Department of Surgery, Faculty of Medicine, University of Medical Sciences and Technology, Sudan \\ *Corresponding author E-mail: awadali82@ hotmail.com
}

\begin{abstract}
Background: Traditional suture repair of inguinal hernia is fast giving way to routine tension-free mesh repair. In many countries, mesh repair is now more common than suture repair.

Objective: To study mesh related complications in the early postoperative period in patient with inguinal hernia.

Methods: This cross-sectional study was conducted at Khartoum teaching hospital. One hundred and three patients who had mesh repair for inguinal hernia were the base for this study between January and December 2011 using a structured questionnaire.

Results: The mean age of the patients is $43.203 \pm 14.41$ years (STD). There were $95.1 \%$ males $(n=98)$ and $4.9 \%$ females ( $n=5)$. Tensionfree mesh repair was done for 103 patients, 12 patients (11.3\%) developed wound hematoma, another 12 patients (11.3\%) developed numbness at the site of operations, 11 patients (10.7\%) developed scrotal swelling, nine patients (8.7\%) developed seroma and four patients $(3.9 \%)$ developed neuralgia at the site of operations.

Conclusion: Mesh repair of inguinal hernias is not free of complications. A larger sample size and further studies are needed to compare outcome of mesh repair in our environment more objectively with traditional inguinal hernia repair.
\end{abstract}

Keywords: Inguinal Hernia; Mesh Repair; Complications.

\section{Introduction}

Inguinal hernia regardless of the type is one of the most common diseases that a surgeon has to manage. Improved surgical techniques and a better understanding of the anatomy and Physiology of the inguinal canal has significantly improves the outcomes for many Patients.

Inguinal hernia repair has been evolving for the past 130 years and the pace of evolution accelerated in the last decade with the introduction of the tension free repair, the laparoscopic repair and the growth of the specialist hernia clinic. (Khan et al. 2008) Traditional suture repair of inguinal hernia is fast giving way to routine tension-free mesh repair. In many countries, mesh repair is now more common than suture repair.(Kaynak et al. 2007) This is mainly because many studies have demonstrated less recurrence with meshes than with traditional suture repair. Furthermore, mesh repair is reported in some studies to reduce operating time and hospital stay.

Lichtenstein presented his open mesh repair technique for inguinal hernia in 1986. The Lichtenstein technique has since become the most commonly used (with various modifications) on account of its ease of operation and because it provides a tension-free repair with good long-term results.(Khan et al. 2006) The advantages of this repair were its association with less pain, rapid postoperative recovery, early return to normal activity and very low recurrence rate.(Bringman et al. 2005) Tension-free mesh repair is nevertheless associated with complications such as foreign body reaction, infection, pain, fistula formation, migration, shrinkage, and recurrence. Other complications include skin anaesthesia, bruising and haematoma formation, seroma formation, orchitis and testicular atrophy. (Agrawal \& Avill 2006) A large number of materials have been tested but currently three are in common use: Polyester mesh (Dacron, Mersilene), Polypropylene (Marlex, Prolene) and Expanded polytetrafluoroethylene (e-PTFE).(Champault et al. 2007)

In this prospective study, we aimed to determine the acceptability, practicality, effectiveness, and safety of inguinal hernia repair using mesh in our environment.

\section{Methods}

This is a cross-sectional study that relied on conducting interviews using a structured questionnaire with a random sample of patients admitted to Khartoum teaching hospital, Khartoum, Sudan. This is a prospective descriptive study performed in Khartoum Teaching Hospital between January and December 2011. One hundred and three patients were included in the study. Patients below 18 years, with chronic constipation, chronic cough; Symptoms of prostatism, Irreducible hernia, obstructed hernia and Strangulated hernia were excluded. A questionnaire designed for this study was used for data collection. A polypropylene mesh was used for all patients who had open hernioplasty. Prophylactic antibiotics were given with induction of anaesthesia in form of second generation cephalosporin (Cefuroxime). Prolene 2/0 suture material was used to fix the mesh to conjoined tendon and inguinal ligament in interrupted manner. Patients were followed for complications for one month postoperatively. 
Data were entered into a Microsoft Excel spreadsheet and the statistical analysis was conducted using SPSS (Version 17). Statistical analysis was carried out using descriptive and analytical statistics. Simple frequencies and cross tabulation were done. Chi square test was used for proportions. Stratification for the patient's sex and educational level was done when relevant. P value of less than 0.05 was considered statistically significant. The consent of the patients was obtained. A full explanation of the purposes and nature of the study was conveyed to them. The potential participants were clearly assured that their participation in this study is voluntary any data obtained would be treated confidentially and for the purpose of the research only.

\section{Results}

Number of patients in this study was 103 patients. Of those patients, there were $95.1 \%$ males $(n=98)$ and $4.9 \%$ females $(n=5)$. Age of the patients ranged from 20-80 years, with mean age of $43.203 \pm 14.41$ years. Seventy five patients $(72.8 \%)$ had right sided inguinal hernia while 24 patients $(23.3 \%)$ had a left sided hernia and 4 patients $(3.9 \%)$ had bilateral hernia. Eighty tow patients
(79.6\%) had indirect hernia and 21 (20.4\%) had direct hernia. Sixty eight patients $(66 \%)$ presented with pure inguinal hernia wile 35 patients $(34 \%)$ presented with inguinoscrotal hernia. $(13.6 \%)(n=14)$ had previous history of surgery for hernia on the same side (recurrent hernia). 12 patients $(12.7 \%)$ were diabetics. Ninety patients $(87.4 \%)$ had spinal anaesthesia, 10 patients $(9.7 \%)$ had general anaesthesia, while $3(2.9 \%)$ had local anaesthesia. All patients received prophylactic antibiotics in a form of second generation cephalosporin (cefuroxime) before the induction of anaesthesia. Seventy six patients $(73.8 \%)$ were operated on by registrars $23(22.3 \%)$ by consultant while 4 patients $(3.9 \%)$ by junior doctors under supervision of registrars or consultants. Duration of surgery was less than $1 \mathrm{hr}$ in $(39.8 \%)$, and more than $1 \mathrm{hr}$ in $(60.2 \%)$. Sixty eight patients $(65 \%)$ stayed in the hospital less than 2 days. Twelve patients $(11.3 \%)$ develops wound hematoma, another 12 patients $(11.3 \%)$ developed numbness at the site of operations, 11patients (10.7\%) developed scrotal swelling, 9 patients (8.7\%) developed seroma and 4 patients (3.9\%) develop neuralgia at the site of operations (Fig.1).

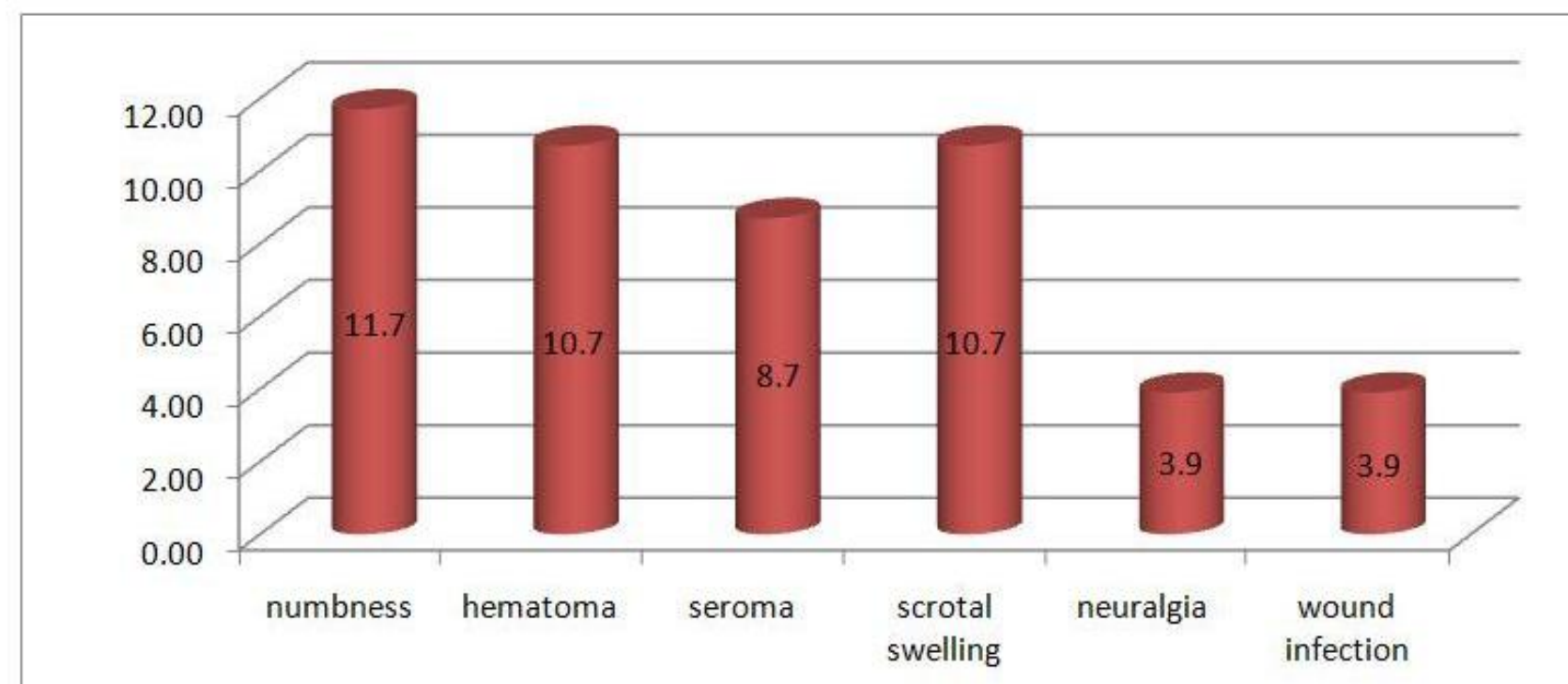

Fig. 1: Showed Incidence of Early Postoperative Complication $(n=103)$

\section{Discussion}

Patients all over the world are happy when they know that their surgery is being performed using a technique that is shown to produce better results than usual with minimal side effects. Patients in this study were no exception, and mesh repair gained immediate acceptance among all in the series. For a century, surgical treatment of inguinal hernia was based on suture repair, but today reinforcement of the groin region by mesh materials is increasingly preferred. Over 1 million meshes per year are implanted for hernia repair worldwide, so successful treatment of groin hernias is of major socioeconomic importance.(Agresta et al. 2010) Hernia has impact on the community by affecting the productive age group. (Buczynowsika et al. 2008) in our study the mean age of patients affected by hernia was $43.203 \pm 14.41$ years which is also productive age group. Inguinal hernia has a male predominance with male to female ratio of 12:1(Buczynowsika et al. 2008) this conforms to our findings were the male represented $95.1 \%$ of the total number of patients enrolled in the study with male to female ratio of 19:1. Those with bilateral hernia were operated for only the side with more symptoms and were given a later date to follow for surgery on the contralateral side. In our study 90 patients had spinal anaesthesia, Spinal anaesthesia provides excellent pain control during herniorrhaphy, and it carries slightly less risk than general anaesthesia. The disadvantages of spinal anaesthesia include the time required for the anaesthetic to be placed and the possibility of incomplete sensory blockade. Urinary retention or a delay in the return of normal lower extremity sensation (Corcione et al. 2005) Of the 4 patients (3.9\%) who developed wound infection, 3 of them developed superficial wound infection that was treated with regular dressing and antibiotics only one patient with deep wound infection treated with removal of mesh. Scrotal swelling occur more common in age group $40-60 \mathrm{yrs}$, all pt with scrotal swelling were treated with analgesia and scrotal support. Scrotal swelling occurs more common in inguinoscrotal hernia around $22 \%(\mathrm{P}=0.004)$ Table (1). Wound hematoma occurs more commonly in age $>60 \mathrm{yrs}$, it occurs more common in patients with recurrent hernia $(\mathrm{P}=0.020)$, all patients with wound hematoma were treated conservatively. Numbness is more common in pt with recurrent inguinal hernia because of higher incidence of nerve injury.

A seroma is a collection of serum in a surgical wound; it contains leukocytes and may also contain some red blood cells. (Farooq \& Bashir ur 2005)The size of the collection relates to the amount of dissection done between tissue planes and the amount of dead space remaining in the wound.(Agarwal et al. 2009) Of 9 patients $(8.7 \%)$ who developed seroma, 5 were treated conservatively and 4 were treated with aspiration under aseptic technique. Severe chronic pain after groin hernia repair is uncommon but potentially debilitating(Aasvang \& Kehlet 2005).Of the 4 patients (3.9\%) develop neuralgia at the site of operations all were treated conser- 
vatively. No patients needed nerve exploration. The cost of meshes is not considered in this study. Also, we do need a larger sample size and further studies for a more objective comparison of outcome between mesh repair and traditional suture repair in our environment.

\section{Conclusions}

Tension-free mesh repair is a simple, safe and effective method of treatment for inguinal hernia with minimal complications. A larger sample size and further studies are needed to compare outcome of mesh repair in our environment more objectively with traditional inguinal hernia repair. Training, experience, and attention to technique will prevent some of these complications.

\section{References}

[1] Aasvang E \& Kehlet H (2005): Surgical management of chronic pain after inguinal hernia repair. $\mathrm{Br} \quad \mathrm{J}$ Surg 92, 795-801. http://dx.doi.org/10.1002/bjs.5103.

[2] Agarwal BB, Agarwal KA \& Mahajan KC (2009): Prospective double-blind randomized controlled study comparing heavy- and lightweight polypropylene mesh in totally extraperitoneal repair of inguinal hernia: early results. Surg Endosc 23, 242-247. http://dx.doi.org/10.1007/s00464-008-0188-2.

[3] Agrawal A \& Avill R (2006): Mesh migration following repair of inguinal hernia: a case report and review of literature. Hernia 10, 7982. http://dx.doi.org/10.1007/s10029-005-0024-8.

[4] Agresta F, Mazzarolo G, Balbi P \& Bedin N (2010): Inguinal-scrotal hernias in young patients: is laparoscopic repair a possible answer? Preliminary results of a single-institution experience with a transabdominal preperitoneal approach. Hernia 14, 471-475. http://dx.doi.org/10.1007/s10029-010-0677-9.

[5] Bringman S, Wollert S, Osterberg J \& Heikkinen T (2005): Early results of a randomized multicenter trial comparing Prolene and VyproII mesh in bilateral endoscopic extraperitoneal hernioplasty (TEP). Surg Endosc 19, 536-540. http://dx.doi.org/10.1007/s00464004-9100-x.

[6] Buczynowsika M, Cienciala A, Friediger J, Steczko-Sieczkowska M, Topa J, Waz K \& Gotfryd-Bugajska K (2008): [Epidemiologic analyse 660 patients operated propter inguinal hernia with implantation synthetic mesh]. Folia Med Cracov 49, 39-44.

[7] Champault G, Bernard C, Rizk N \& Polliand C (2007): Inguinal hernia repair: the choice of prosthesis outweighs that of technique Hernia 11, 125-128. http://dx.doi.org/10.1007/s10029-006-0179-y.

[8] Corcione F, Pede A, Cuccurullo D, Marzano E, Manzi F, Pirozzi F \& Ruggiero R (2005): Treatment of primary inguinal hernias by "held in mesh repair": our experience related to 3,520 cases. Hernia 9, 263268. http://dx.doi.org/10.1007/s10029-005-0329-7.

[9] Farooq O \& Bashir ur R (2005): Recurrent inguinal hernia repair by open preperitoneal approach. J Coll Physicians Surg Pak 15, 261-265.

[10]Kaynak B, Celik F, Guner A, Guler K, Kaya MA \& Celik M (2007) Moloney darn repair versus lichtenstein mesh hernioplasty for open inguinal hernia repair. Surg Today 37, 958-960. http://dx.doi.org/10.1007/s00595-007-3543-3.

[11]Khan LR, Kumar S \& Nixon SJ (2006): Early results for new lightweight mesh in laparoscopic totally extra-peritoneal inguinal hernia repair. Hernia 10, 303-308. http://dx.doi.org/10.1007/s10029-0060093-3.

[12]Khan N, Naeem M, Bangash A, Asadullah, Sadiq M \& Hamid H (2008): Early outcome of Lichtenstein technique of tension-free open mesh repair for inguinal hernia. J Ayub Med Coll Abbottabad 20, 29 